\title{
A new method to choose the approximate function in order to determine the correlation between the imposed and the obtained clearances in FDM
}

\author{
Maria-Luiza BEȘLIU-GHERGHESCU ${ }^{1}$, Nicolae-Doru STĂNESCU ${ }^{1, *}$ \\ ${ }^{1}$ University of Pitești, Romania, \\ *Corresponding author e-mail: doru.stanescu@upit.ro \\ Article history \\ Received 15.08.2020 \\ Accepted 25.09.2020 \\ DOI https://doi.org/10.26825/bup.ar.2020.004
}

\begin{abstract}
Practical experiments proved that the variation of clearance between two circular pieces simultaneously manufactured by FDM, in function of different parameters has a complex variation starting from zero when the imposed clearance has a small value and being approximated by the value of the imposed clearance when the last one has great values. This observation implies that the resulted clearance may be approximated by non-linear functions for which one has to impose some frontier conditions. In this paper we discuss the conditions which have to be fulfilled by the candidate functions, considered only by polynomial ones.
\end{abstract}

Keywords: Fused Deposition Modelling, clearance, polynomial candidate functions, frontier conditions

\section{Introduction}

The variations between different parameters are usually considered by using the Least Square Method [1]. This method has the advantage of simplicity and it is easily implemented. Most books and papers treat the case of one input and one output variable, while the approximation is obtained as a polynomial one. The great problem is that this method and its results do not generally satisfy the frontier conditions. Some particular methods are developed in the case of manufacturing, optimization and FDM.

Rozvany [2] uses exponential penalty functions in order to obtain topological optimizations. Other authors [3] use some weight functions in order to determine some indices of the FDM processes. Jin et al. [4] describe the path followed by the tool in the case of complex surfaces and introduce supplementary control points in order to be fulfilled some frontier conditions. In [5] the authors use the minimization of a linear combination of quadratic functions for the solving of the problems of topological optimization. Langelaar [6] uses logarithmic functions for the topological optimization. In [7] the authors start from the heat-transfer equation and use the rheological Carreau model to construct a numerical model to improve the FDM process. Garg and Bhattacharya [8] use the finite element method to analyze the failure of the FDM parts to tensile loading. Ponce and Kerbrat [9] solve the heat-transfer equation using the finite element method using polynomial expressions for the frontier conditions. Mohamed et al. use a regression quadratic model with six factor model and the ANOVA technique in order to determine the correlations between the input and output variables.

The references show that the authors use different expressions of functions in order to obtain better approximations of the correlation functions. 
Considering the clearance obtained by FDM process, we have to state that any function which would be tried has to satisfy some frontier conditions: for small imposed clearance the practical results prove that the clearance obtained by FDM vanishes. Moreover, there exists a limit value from which the obtained clearance has non-zero value. This means that the expression of the obtained clearance must vanish if the imposed one is smaller than a value, let us say $a$. Because the obtained clearance does not start to increase abruptly, we have to impose the condition that the derivative of the expression of the obtained clearance must vanish at the same point. Practical experiments prove that if the imposed clearance is sufficiently high, the obtained clearance is equal to the imposed one. It results that there exists a limit, let it be $b$, above which the two values (the imposed clearance and the obtained one) coincide. In addition, the derivative of the expression of the obtained clearance must be equal to unity at the same point. These conditions, which we call by frontier conditions, limit the possibility of choosing the expressions of the desired correlation functions.

The paper is organized as follows. In the second part we consider the case of one input parameter. Here we also discuss the possibility of choosing particular rational functions. The third part is dedicated to the case of two input parameters, while the fourth one treats the situation of four input candidates. The paper ends with conclusions.

\section{The case of one parameter}

\subsection{Scaling procedure}

Let us consider the interval $[0,1]$ and the polynomial function $f: \mathbf{R} \rightarrow \mathbf{R}$.

The imposed conditions are

$$
f(0)=0, f(1)=1, f^{\prime}(0)=0, f^{\prime}(1)=1,
$$

totally four conditions; it implies that the polynomial function has the degree equal to three,

$$
f(x)=a_{3} x^{3}+a_{2} x^{2}+a_{1} x+a_{0} .
$$

The four conditions lead to the linear system

$$
a_{0}=0, a_{1}=0, a_{3}+a_{2}=1,3 a_{3}+2 a_{2}=1,
$$

wherefrom

$$
a_{3}=-1, a_{2}=2
$$

and the function and its derivative

$$
f(x)=-x^{3}+2 x^{2}, f^{\prime}(x)=-3 x^{2}+4 x .
$$

We pass now to the interval $[a, b]$. The passing from the interval $[0,1]$ to the interval $[a, b]$ is performed with the aid of the system

$$
\alpha a+\beta=0, \alpha b+\beta=1,
$$

that is

$$
\alpha=\frac{1}{b-a}, \beta=\frac{a}{b-a}
$$

hence

$$
f_{1}(x)=-\frac{(x-a)^{3}}{(b-a)^{3}}+2 \frac{(x-a)^{2}}{(b-a)^{2}}, f_{1}^{\prime}(x)=-3 \frac{(x-a)^{2}}{(b-a)^{3}}+4 \frac{x-a}{(b-a)^{2}}
$$

It results 


$$
f_{1}(a)=0, f_{1}(b)=1, f_{1}^{\prime}(a)=0, f_{1}^{\prime}(b)=\frac{1}{b-a} .
$$

In conclusion, the scaling procedure does not work in this situation, and we have to consider directly the interval $[a, b]$.

\subsection{General interval}

In this situation the conditions are

$$
f(a)=0, f(b)=v, f^{\prime}(a)=0, f^{\prime}(b)=1,
$$

where $v$ is a positive real value.

The explanation for the condition $f^{\prime}(b)=1$ is as follows: when the imposed clearance is sufficiently high, then the obtained clearance is equal to the imposed one.

Writing

$$
f_{2}(x)=a_{3} x^{3}+a_{2} x^{2}+a_{1} x+a_{0}, f^{\prime}(x)=3 a_{3} x^{2}+2 a_{2} x+a_{1},
$$

one deduces the linear system of four equations with four unknowns

$$
\begin{gathered}
a_{3} a^{3}+a_{2} a^{2}+a_{1} a+a_{0}=0, \\
a_{3} b^{3}+a_{2} b^{2}+a_{1} b+a_{0}=v, 3 a_{3} a^{2}+2 a_{2} a+a_{1}=0, \\
3 a_{3} b^{2}+2 a_{2} b+a_{1}=1 .
\end{gathered}
$$

The determinant of the system reads

$$
\begin{aligned}
\Delta & =\left|\begin{array}{llll}
a^{3} & a^{2} & a & 1 \\
b^{3} & b^{2} & b & 1 \\
3 a^{2} & 2 a & 1 & 0 \\
3 b^{2} & 2 b & 1 & 0
\end{array}\right|=\left|\begin{array}{cccc}
a^{3} & a^{2} & a & 1 \\
b^{3}-a^{3} & b^{2}-a^{2} & b-a & 0 \\
3 a^{2} & 2 a & 1 & 0 \\
3 b^{2} & 2 b & 1 & 0
\end{array}\right| \\
& =-\left|\begin{array}{ccc}
b^{3}-a^{3} & b^{2}-a^{2} & b-a \\
3 a^{2} & 2 a & 1 \\
3 b^{2} & 2 b & 1
\end{array}\right|=-(b-a)^{4} \neq 0,
\end{aligned}
$$

that is, the system has a unique solution.

Moreover,

$$
\begin{aligned}
\Delta_{3} & =\left|\begin{array}{llll}
0 & a^{2} & a & 1 \\
v & b^{2} & b & 1 \\
0 & 2 a & 1 & 0 \\
1 & 2 b & 1 & 0
\end{array}\right|=\left|\begin{array}{cccc}
0 & a^{2} & a & 1 \\
v & b^{2}-a^{2} & b-a & 0 \\
0 & 2 a & 1 & 0 \\
1 & 2 b & 1 & 0
\end{array}\right| \\
& =\left|\begin{array}{ccc}
v & b^{2}-a^{2} & b-a \\
0 & 2 a & 1 \\
1 & 2 b & 1
\end{array}\right|=(b-a)(2 v-b+a),
\end{aligned}
$$




$$
\begin{aligned}
& \Delta_{1}=\left|\begin{array}{cccc}
a^{3} & a^{2} & 0 & 1 \\
b^{3} & b^{2} & v & 1 \\
3 a^{2} & 2 a & 0 & 0 \\
3 b^{2} & 2 b & 1 & 0
\end{array}\right|=\left|\begin{array}{cccc}
a^{3} & a^{2} & 0 & 1 \\
b^{3}-a^{3} & b^{2}-a^{2} & v & 0 \\
3 a^{2} & 2 a & 0 & 0 \\
3 b^{2} & 2 b & 1 & 0
\end{array}\right| \\
& =-\left|\begin{array}{ccc}
b^{3}-a^{3} & b^{2}-a^{2} & v \\
3 a^{2} & 2 a & 0 \\
3 b^{2} & 2 b & 1
\end{array}\right|=(b-a)\left(6 a b v+a^{3}+a^{2} b-2 a b^{2}\right) \text {, } \\
& \Delta_{0}=\left|\begin{array}{cccc}
a^{3} & a^{2} & a & 0 \\
b^{3} & b^{2} & b & v \\
3 a^{2} & 2 a & 1 & 0 \\
3 b^{2} & 2 b & 1 & 1
\end{array}\right|=v\left|\begin{array}{ccc}
a^{3} & a^{2} & a \\
3 a^{2} & 2 a & 1 \\
3 b^{2} & 2 b & 1
\end{array}\right|+\left|\begin{array}{ccc}
a^{3} & a^{2} & a \\
b^{3} & b^{2} & b \\
3 a^{2} & 2 a & 1
\end{array}\right| \\
& =a^{2}(b-a)\left[v(a-3 b)+b^{2}-a b\right]
\end{aligned}
$$

and one gets $a_{3}=\frac{\Delta_{3}}{\Delta}, a_{2}=\frac{\Delta_{2}}{\Delta}, a_{1}=\frac{\Delta_{1}}{\Delta}, a_{0}=\frac{\Delta_{0}}{\Delta}$.

For instance, if $a=0, b=1$ and $v=1$, it results

$$
\begin{gathered}
\Delta=-1, \Delta_{3}=1, \Delta_{2}=-2, \Delta_{1}=0, \Delta_{0}=0, \\
f(x)=-x^{3}+2 x^{2} .
\end{gathered}
$$

\subsection{General candidates}

\subsubsection{Polynomial function}

One may choose

$$
f(x)=a_{n} x^{n}+a_{n-1} x^{n-1}+\ldots+a_{1} x+a_{0},
$$

wherefrom

$$
f^{\prime}(x)=n a_{n} x^{n-1}+(n-1) a_{n-1} x^{n-2}+\ldots+a_{1},
$$

The conditions (10) lead to

$$
\begin{gathered}
f(a)=a_{n} a^{n}+a_{n-1} a^{n-1}+\ldots+a_{1} a+a_{0}=0, f(b)=a_{n} b^{n}+a_{n-1} b^{n-1}+\ldots+a_{1} b+a_{0}=v, \\
f^{\prime}(a)=n a_{n} a^{n-1}+(n-1) a_{n-1} a^{n-1}+\ldots+a_{1}=0, f^{\prime}(b)=n a_{n} b^{n-1}+(n-1) a_{n-1} b^{n-1}+\ldots+a_{1}=1,
\end{gathered}
$$

which is a linear system with four equations and $n+1$ unknowns $\left(a_{n}, a_{n-1}, \ldots, a_{0}\right)$; this system is a compatible and non-determined one.

\subsubsection{First rational function}

Another possibility for the function $f$ is a rational one. Taking into account that we have four constraints, the rational function $f$ may be written as

$$
f(x)=\frac{c_{1} x+c_{2}}{d_{1} x+d_{2}}, f^{\prime}(x)=\frac{c_{1} d_{2}-c_{2} d_{1}}{\left(d_{1} x+d_{2}\right)^{2}} .
$$

Limiting the conditions to the interval $[0,1]$ one gets the relations 


$$
f(0)=\frac{c_{2}}{d_{2}}=0
$$

wherefrom $c_{2}=0$,

$$
f^{\prime}(0)=\frac{c_{1} d_{2}-c_{2} d_{1}}{d_{2}^{2}}=0
$$

with $c_{1}=0$; that is

$$
f(x)=\frac{0}{d_{1} x}=0 ; f(0)=0, f(1)=0,
$$

which is impossible.

\subsubsection{Second rational function}

Choosing now

$$
f(x)=\frac{c_{1} x^{2}+c_{2} x+c_{3}}{d_{1} x+d_{2}}, f^{\prime}(x)=\frac{c_{1} d_{1} x^{2}+2 c_{1} d_{2} x-c_{3} d_{1}}{\left(d_{1} x+d_{2}\right)^{2}},
$$

with the same conditions, one obtains

$$
\begin{gathered}
f(0)=\frac{c_{3}}{d_{2}}=0, c_{3}=0, \\
f^{\prime}(0)=-\frac{c_{3} d_{1}}{d_{2}}=0, \\
f(1)=\frac{c_{1}+c_{2}}{d_{1}+d_{2}}=1, \\
f^{\prime}(1)=\frac{c_{1} d_{1}+2 c_{1} d_{2}}{d_{1}+d_{2}}=1 .
\end{gathered}
$$

The last two relations form a nonlinear system of two equations with four unknowns $\left(c_{1}, c_{2}, d_{1}, d_{2}\right)$, which, in general, has an infinity of solutions

\section{Case of two parameters}

\subsection{First choice}

One may select

$$
\begin{gathered}
f(x, y)=\left(a_{2} x^{2}+a_{1} x+a_{0}\right)\left(\mathrm{b}_{2} y^{2}+b_{1} y+b_{0}\right), \\
\frac{\partial f}{\partial x}=\left(2 a_{2} x+a_{1}\right)\left(\mathrm{b}_{2} y^{2}+b_{1} y+b_{0}\right),
\end{gathered}
$$




$$
\frac{\partial f}{\partial y}=\left(a_{2} x^{2}+a_{1} x+a_{0}\right)\left(2 \mathrm{~b}_{2} y+b_{1}\right) .
$$

Considering the same interval $[a, b]$ the new conditions are

$$
\begin{gathered}
f(a, a)=0, f(b, b)=v, \\
\frac{\partial f}{\partial x}(a, a)=0, \frac{\partial f}{\partial y}(a, a)=0, \\
\frac{\partial f}{\partial x}(b, b)=1, \frac{\partial f}{\partial y}(b, b)=1,
\end{gathered}
$$

which offer the system

$$
\begin{gathered}
\left(a_{2} a^{2}+a_{1} a+a_{0}\right)\left(\mathrm{b}_{2} a^{2}+b_{1} a+b_{0}\right)=0,\left(a_{2} b^{2}+a_{1} b+a_{0}\right)\left(\mathrm{b}_{2} b^{2}+b_{1} b+b_{0}\right)=v, \\
\left(2 a_{2} a+a_{1}\right)\left(\mathrm{b}_{2} a^{2}+b_{1} a+b_{0}\right)=0,\left(a_{2} a^{2}+a_{1} a+a_{0}\right)\left(2 \mathrm{~b}_{2} a+b_{1}\right)=0, \\
\left(2 a_{2} b+a_{1}\right)\left(\mathrm{b}_{2} b^{2}+b_{1} b+b_{0}\right)=1,\left(a_{2} b^{2}+a_{1} b+a_{0}\right)\left(2 \mathrm{~b}_{2} b+b_{1}\right)=1 .
\end{gathered}
$$

From the first equation (38) one gets either

$$
a_{2} a^{2}+a_{1} a+a_{0}=0, b_{2} a^{2}+b_{1} a+b_{0} \neq 0
$$

or

$$
b_{2} a^{2}+b_{1} a+b_{0}=0, a_{2} a^{2}+a_{1} a+a_{0} \neq 0
$$

or

$$
a_{2} a^{2}+a_{1} a+a_{0}=0, b_{2} a^{2}+b_{1} a+b_{0}=0 .
$$

Let us consider the case (41). It results that the third, fourth, fifth and sixth equations (38) are useless. One obtains the system

$$
a_{2} a^{2}+a_{1} a+a_{0}=0, b_{2} a^{2}+b_{1} a+b_{0}=0,\left(a_{2} b^{2}+a_{1} b+a_{0}\right)\left(b_{2} b^{2}+b_{1} b+b_{0}\right)=v,
$$

which is a nonlinear system of three equations with six unknowns $\left(a_{2}, a_{1}, a_{0}, b_{2}, b_{1}, b_{0}\right)$; in general, the system is a non-determined one.

If we choose the variant (39), we obtain that the fourth equation (38) is useless, while the third equation (38) leads to

$$
b_{2} a^{2}+b_{1} a+b_{0}=0 \text {, }
$$

which is a contradiction with the second assumption (39).

One may similarly discuss the case (40).

\subsection{Second choice}

Let us consider the function

$$
f(x, y)=a_{20} x^{2}+a_{11} x+a_{02} y^{2}+a_{10} x+a_{01} y+a_{00},
$$

for which 


$$
\begin{aligned}
& \frac{\partial f}{\partial x}=2 a_{20} x+a_{11} y+a_{10}, \\
& \frac{\partial f}{\partial y}=a_{11} x+2 a_{02} y+a_{01} .
\end{aligned}
$$

Working with the same conditions (35), (36), (37), it results the equations

$$
\begin{aligned}
f(a, a)= & a_{20} a^{2}+a_{11} a^{2}+a_{02} a^{2}+a_{10} a+a_{01} a+a_{00}=0, \\
f(b, b)= & a_{20} b^{2}+a_{11} b^{2}+a_{02} b^{2}+a_{10} b+a_{01} b+a_{00}=v, \\
& \frac{\partial f}{\partial x}(a, a)=2 a_{20} a+a_{11} a+a_{10}=0, \\
& \frac{\partial f}{\partial y}(a, a)=a_{11} a+2 a_{02} a+a_{01}=0, \\
& \frac{\partial f}{\partial x}(b, b)=2 a_{20} b+a_{11} b+a_{10}=1, \\
\frac{\partial f}{\partial y}(b, b)= & a_{11} b+2 a_{02} b+a_{01}=1 .
\end{aligned}
$$

These equations form a linear system of six equations with six unknowns $\left(a_{2}, a_{1}, a_{0}, b_{2}, b_{1}, b_{0}\right)$, the determinant of which being

$$
\Delta=\left|\begin{array}{cccccc}
a^{2} & a^{2} & a^{2} & a & a & 1 \\
b^{2} & b^{2} & b^{2} & b & b & 1 \\
2 a & a & 0 & 1 & 0 & 0 \\
0 & a & 2 a & 0 & 1 & 0 \\
2 b & b & 0 & 1 & 0 & 0 \\
0 & b & 2 b & 0 & 1 & 0
\end{array}\right| .
$$

The determinant may be successively written

$$
\Delta=\left|\begin{array}{cccccc}
a^{2} & a^{2} & a^{2} & a & a & 1 \\
b^{2}-a^{2} & b^{2}-a^{2} & b^{2}-a^{2} & b-a & b-a & 0 \\
2 a & a & 0 & 1 & 0 & 0 \\
0 & a & 2 a & 0 & 1 & 0 \\
2 b & b & 0 & 1 & 0 & 0 \\
0 & b & 2 b & 0 & 1 & 0
\end{array}\right|,
$$




$$
\begin{aligned}
& \Delta=(a-b)\left|\begin{array}{ccccc}
a+b & a+b & a+b & -1 & -1 \\
2 a & a & 0 & 1 & 0 \\
0 & a & 2 a & 0 & 1 \\
2 b & b & 0 & 1 & 0 \\
0 & b & 2 b & 0 & 1
\end{array}\right| \\
& \Delta=(a-b)\left|\begin{array}{ccccc}
a+b & a+b & a+b & -1 & -1 \\
2 a & a & 0 & 1 & 0 \\
a+b & 2 a+b & 3 a+b & -1 & 0 \\
2 b & b & 0 & 1 & 0 \\
0 & a+2 b & a+3 b & 0 & 0
\end{array}\right|, \\
& \Delta=(b-a)\left|\begin{array}{cccc}
2 a & a & 0 & 1 \\
a+b & 2 a+b & 3 a+b & -1 \\
2 b & b & 0 & 1 \\
0 & a+2 b & a+3 b & 0
\end{array}\right|, \\
& \Delta=(b-a)\left|\begin{array}{cccc}
2 a & a & 0 & 1 \\
3 a+b & 3 a+b & 3 a+b & 0 \\
2 b-2 a & b-a & 0 & 0 \\
0 & a+2 b & a+3 b & 0
\end{array}\right| \\
& \Delta=(a-b)(3 a+b)(b-a)\left|\begin{array}{ccc}
1 & 1 & 1 \\
2 & 1 & 0 \\
0 & a+2 b & a+3 b
\end{array}\right|=-(b-a)^{2}(3 a+b)(a+b) \neq 0,
\end{aligned}
$$

that is the system has a unique solution.

\section{Case of three parameters}

In this situation the conditions for the function $f(x, y, z)$ are as follows:

$$
\begin{gathered}
f(a, a, a)=0, f(b, b, b)=v, \\
\frac{\partial f}{\partial x}(a, a, a)=0, \frac{\partial f}{\partial y}(a, a, a)=0, \frac{\partial f}{\partial z}(a, a, a)=0, \\
\frac{\partial f}{\partial x}(b, b, b)=1, \frac{\partial f}{\partial y}(b, b, b)=1, \frac{\partial f}{\partial z}(b, b, b)=1,
\end{gathered}
$$

that is a number of six conditions.

\subsection{First candidate}

We select as function $f$ the expression 


$$
f(x, y, z)=\left(a_{1} x+a_{2}\right)\left(b_{1} y+b_{2}\right)\left(c_{1} z+c_{2}\right),
$$

with

$$
\begin{aligned}
& \frac{\partial f}{\partial x}=a_{1}\left(b_{1} y+b_{2}\right)\left(c_{1} z+c_{2}\right), \\
& \frac{\partial f}{\partial y}=\left(a_{1} x+a_{2}\right) b_{1}\left(c_{1} z+c_{2}\right), \\
& \frac{\partial f}{\partial z}=\left(a_{1} x+a_{2}\right)\left(b_{1} y+b_{2}\right) c_{1},
\end{aligned}
$$

The conditions (60), (61), (62) leads to

$$
\begin{gathered}
f(a, a, a)=\left(a_{1} a+a_{2}\right)\left(b_{1} a+b_{2}\right)\left(c_{1} a+c_{2}\right)=0, f(b, b, b)=\left(a_{1} b+a_{2}\right)\left(b_{1} b+b_{2}\right)\left(c_{1} b+c_{2}\right)=v, \\
\frac{\partial f}{\partial x}(a, a, a)=a_{1}\left(b_{1} a+b_{2}\right)\left(c_{1} a+c_{2}\right)=0, \frac{\partial f}{\partial y}(a, a, a)=\left(a_{1} a+a_{2}\right) b_{1}\left(c_{1} a+c_{2}\right)=0, \\
\frac{\partial f}{\partial z}(a, a, a)=\left(a_{1} a+a_{2}\right)\left(b_{1} a+b_{2}\right) c_{1}=0, \frac{\partial f}{\partial x}(b, b, b)=a_{1}\left(b_{1} b+b_{2}\right)\left(c_{1} b+c_{2}\right)=1, \\
\frac{\partial f}{\partial y}(b, b, b)=\left(a_{1} b+a_{2}\right) b_{1}\left(c_{1} b+c_{2}\right)=1, \frac{\partial f}{\partial z}(b, b, b)=\left(a_{1} b+a_{2}\right)\left(b_{1} b+b_{2}\right) c_{1}=1 .
\end{gathered}
$$

First expression (67) implies either

$$
a_{1} a+a_{2}=0
$$

or

$$
b_{1} a+b_{2}=0
$$

or

$$
c_{1} a+c_{2}=0 .
$$

Let us assume that the relation (68) holds true.

From the third relation (67) it results that either

$$
b_{1} a+b_{2}=0
$$

or

$$
c_{1} a+c_{2}=0 .
$$

We assume that the expression (71) is the valid one.

One thus obtains

$$
\begin{gathered}
a_{1} a+a_{2}=0, a_{2}=-a_{1} a, \\
b_{1} a+b_{2}=0, b_{2}=-b_{1} a, \\
f(x, y, z)=a_{1} b_{1}(x-a)(y-a)\left(c_{1} z+c_{2}\right)
\end{gathered}
$$

The sixth and seventh equations (67) offer

$$
a_{1} b_{1}(b-a)\left(c_{1} b+c_{2}\right)=1,
$$

wherefrom 


$$
a_{1} b_{1}=\frac{1}{(b-a)\left(c_{1} b+c_{2}\right)} \text {. }
$$

The eight expression (67) gives

$$
a_{1} b_{1}(b-a)^{2} c_{1}=1,
$$

that is,

$$
\frac{b-a}{c_{1} b+c_{2}} c_{1}=1,
$$

wherefrom

$$
\begin{gathered}
c_{2}=-a c_{1}, \\
f(x, y, z)=a_{1} b_{1} c_{1}(x-a)(y-a)(z-a) .
\end{gathered}
$$

In conclusion

$$
a_{1} b_{1} c_{1}(b-a)^{2}=1
$$

and

$$
a_{1} b_{1} c_{1}(b-a)^{3}=v ;
$$

it results two supplementary conditions

$$
v=b-a
$$

and

$$
a_{1} b_{1} c_{1}=\frac{1}{(b-a)^{2}},
$$

that is, the function $f(x, y, z)$ given by (63) may be used only in same particular conditions.

\subsection{Second candidate}

Selecting now

$$
f(x, y, z)=\left(a_{2} x^{2}+a_{1} x+a_{0}\right)\left(b_{2} y^{2}+b_{1} y+b_{0}\right)\left(c_{2} z^{2}+c_{1} z+c_{0}\right)
$$

for which

$$
\begin{aligned}
& \frac{\partial f}{\partial x}=\left(2 a_{2} x+a_{1}\right)\left(b_{2} y^{2}+b_{1} y+b_{0}\right)\left(c_{2} z^{2}+c_{1} z+c_{0}\right) \\
& \frac{\partial f}{\partial y}=\left(a_{2} x^{2}+a_{1} x+a_{0}\right)\left(2 b_{2} y+b_{1}\right)\left(c_{2} z^{2}+c_{1} z+c_{0}\right) \\
& \frac{\partial f}{\partial z}=\left(a_{2} x^{2}+a_{1} x+a_{0}\right)\left(b_{2} y^{2}+b_{1} y+b_{0}\right)\left(2 c_{2} z+c_{1}\right)
\end{aligned}
$$

and the frontier conditions $(60),(61),(62)$ it results the system

$$
\left(a_{2} a^{2}+a_{1} a+a_{0}\right)\left(b_{2} a^{2}+b_{1} a+b_{0}\right)\left(c_{2} a^{2}+c_{1} a+c_{0}\right)=0,
$$




$$
\begin{gathered}
\left(a_{2} b^{2}+a_{1} b+a_{0}\right)\left(b_{2} b^{2}+b_{1} b+b_{0}\right)\left(c_{2} b^{2}+c_{1} b+c_{0}\right)=v, \\
\left(2 a_{2} a+a_{1}\right)\left(b_{2} a^{2}+b_{1} a+b_{0}\right)\left(c_{2} a^{2}+c_{1} a+c_{0}\right)=0,\left(2 b_{2} a+b_{1}\right)\left(a_{2} a^{2}+a_{1} a+a_{0}\right)\left(c_{2} a^{2}+c_{1} a+c_{0}\right)=0, \\
\left(2 c_{2} a+c_{1}\right)\left(a_{2} a^{2}+a_{1} a+a_{0}\right)\left(b_{2} a^{2}+b_{1} a+b_{0}\right)=0,\left(2 a_{2} b+a_{1}\right)\left(b_{2} b^{2}+b_{1} b+b_{0}\right)\left(c_{2} b^{2}+c_{1} b+c_{0}\right)=1, \\
\left(2 b_{2} b+b_{1}\right)\left(a_{2} b^{2}+a_{1} b+a_{0}\right)\left(c_{2} b^{2}+c_{1} b+c_{0}\right)=1,\left(2 c_{2} b+c_{1}\right)\left(a_{2} b^{2}+a_{1} b+a_{0}\right)\left(b_{2} b^{2}+b_{1} b+b_{0}\right)=1 .
\end{gathered}
$$

The first equation (90) leads to either

$$
a_{2} a^{2}+a_{1} a+a_{0}=0
$$

or

$$
b_{2} a^{2}+b_{1} a+b_{0}=0
$$

or

$$
c_{2} a^{2}+c_{1} a+c_{0}=0 .
$$

Let us assume that the expression (91) is valid.

From the third relation (90) one gets the expression (92) or (93) and we assume that (92) holds true.

Moreover, the fourth and fifth expression (90) are useless. One has now only six conditions with nine unknowns.

Taking into account the second, sixth, seventh and eighth relations (93), one obtains the expressions

$$
\begin{aligned}
& \frac{a_{2} b^{2}+a_{1} b+a_{0}}{2 a_{2}+a_{1}}=v, \\
& \frac{b_{2} b^{2}+b_{1} b+b_{0}}{2 b_{2}+b_{1}}=v, \\
& \frac{c_{2} b^{2}+c_{1} b+v_{0}}{2 c_{2}+c_{1}}=v,
\end{aligned}
$$

that is, a number of three supplementary conditions.

If one assumes that the equation (94) is true, then one deduces

$$
a_{2} b^{2}+a_{1} b+a_{0}=2 a_{2} v+a_{1} v .
$$

It results the system

$$
\begin{gathered}
a_{2}\left(b^{2}-2 v\right)+a_{1}(b-v)+a_{0}=0, \\
a_{2} a^{2}+a_{1} a+a_{0}=0 .
\end{gathered}
$$

which is a linear system of two equations with three unknowns $\left(a_{2}, a_{1}, a_{0}\right)$.

Generally, this system has an infinity of solutions.

\section{CONCLUSIONS}

This paper deals with the selection of some candidate functions which has to fulfill some frontier conditions. As we saw, the frontier conditions impose some particular polynomial candidate functions. The selection may be not always possible, but the reader may consider higher degrees polynomials, case in which the problem may lead to a infinite number of solution. 
Let us observe that the functions which respect the frontier conditions may not respect the optimization criterion given by the Last Square Method (the error function is minimum).

In a future paper we will try to add some optimization criterion / criteria to the method of selection of the candidate functions described in the present work.

\section{References}

[1] Teodorescu, P., P., Stănescu, N.-D., Pandrea, N. (2013). Numerical Analysis with Applications in Mechanics and Engineering. John Wiley \& Sons, Hoboken, USA.

[2] Rozvany, G., I., N. (2009) A critical review of established methods of structural topology optimization, Structural and Multidisciplinary Optimization, 37, 217-237.

[3] Xue, D., Imaniyan, D. (2018). A framework for optimal design of complex products. Proceedia CIRP, 70, 416-421.

[4] Jin, G., Q., Li, W., D., Tsai, C., F., Wang, L. (2011). Adaptive tool-path generation of rapid prototyping for complex product models. Journal of Manufacturing Systems, 30, 154-164.

[5] Primo, T., Calabrese, M., Del Prete, A., Anglani, A. (2017). Additive manufacturing integration with topology optimization methodology for innovative product design. International Journal of Advanced Manufacturing Technology, 93, 467-479.

[6] Langelaar, M. (2017). An additive manufacturing filter for topology optimization of print-ready designs. Structural and Multidisciplinary Optimization, 55, 871-883.

[7] Jun, D., Zhengying, W., Xin, W., Jiji, W., Zhen, C. (2016). An improved fused deposition modeling process for formingl arge-size thin-walled parts. Journal of Materials Processing Technology, 234, $332-341$.

[8] Garg, A., Bhattacharya, A. (2017). An Insight to the Failure of FDM Parts under Tensile Loading: Finite Element Analysis and Experimental Study. International Journal of Mechanical Sciences, 120, 225-236.

[9] Ponchen, R., Kerbrat, O., Mognol, P., Hascoet, J.-Y. (2014). A novel methodology of design for Additive Manufacturing applied to Additive Laser Manufacturing process. Robotics and ComputerIntegrated Manufacturing, 30, 389-398.

[10] Mohamed, O., A., Masood, S., H., Bhowmik, J., L. (2017). Characterization and dynamic mechanical analysis of PC-ABS material processed by fused deposition modelling: An investigation through I-optimal response surface methodology. Measurement 107, 128-141. 